\title{
Research Article \\ Effects of the Rho-Kinase Inhibitor Y-27632 on Extraocular Muscle Surgery in Rabbits
}

\author{
Ji-Sun Moon, ${ }^{1}$ Hyun Kyung Kim, ${ }^{2}$ and Sun Young Shin ${ }^{1}$ \\ ${ }^{1}$ Department of Ophthalmology and Visual Science, Seoul St. Mary's Hospital, College of Medicine, The Catholic University of Korea, \\ No. 222 Banpo-daero, Seocho-gu, Seoul 06591, Republic of Korea \\ ${ }^{2}$ Department of Ophthalmology, St. Vincent's Hospital, College of Medicine, The Catholic University of Korea, No. 93 Jungbu-daero, \\ Paldal-gu, Suwon 16247, Republic of Korea
}

Correspondence should be addressed to Sun Young Shin; eyeshin@catholic.ac.kr

Received 2 March 2017; Revised 27 May 2017; Accepted 15 June 2017; Published 26 July 2017

Academic Editor: Naoki Okumura

Copyright (C) 2017 Ji-Sun Moon et al. This is an open access article distributed under the Creative Commons Attribution License, which permits unrestricted use, distribution, and reproduction in any medium, provided the original work is properly cited.

Purpose. To evaluate the effect of the Rho-kinase inhibitor Y-27632 on postoperative inflammation and adhesion following extraocular muscle surgery in rabbits. Methods. The superior rectus muscle reinsertion was performed on both eyes of 8 New Zealand white rabbits. After reinsertion, the rabbits received subconjunctival injections of the Rho-kinase inhibitor and saline on each eye. To assess acute and late inflammatory changes, Ki-67, CD11 $\beta+$, and F4/80 were evaluated and the sites of muscle reattachment were evaluated for a postoperative adhesion score and histopathologically for collagen formation. Results. F4/80 antibody expression was significantly different in the Rho-kinase inhibitor-injected group at both postoperative day 3 and week $4(p=0.038,0.031)$. However, Ki-67 and CD11 $\beta+$ were not different the between two groups. The difference in the SRM/conjunctiva adhesion score between the two groups was also significant $(p=0.034)$. Conclusion. Intraoperative subconjunctival injection of the Rho-kinase inhibitor may be effective for adjunctive management of inflammation and fibrosis in rabbit eyes following extraocular muscle surgery.

\section{Introduction}

During the surgical wound healing after extraocular muscle surgery, the damaged tissue must restore its original function and structural integrity. Therefore, it is important to prevent inflammation and fibrosis, as the perfect restoration of previous tissue architecture without scar is the purpose of wound healing. The major complications of strabismus surgery are postoperative adhesions and fibrosis, which can cause limitation of ocular movement and adversely affect the surgical outcome. These adhesions can occur with various tissues, extraocular muscles, sclera, orbital fat, intermuscular membrane, Tenon's capsule, and conjunctiva.

Numerous studies by strabismologists have been devoted to reducing postoperative adhesions using a variety of materials, including polyglactin 910 mesh [1], mitomycin C $[2,3]$, and Seprafilm (Genzyme, Cambridge, MA) [4]. However, these methods have problems with their associated complications, unavailability, or inconsistent results. There is also a debate as to whether mitomycin $\mathrm{C}$ reduces inflammatory response after strabismus surgery significantly $[2,3]$.

Small guanosine triphosphatase (GTPase) Rho-kinase, a member of the Rho-kinase subfamily of the Ras superfamily of monomeric GTPases, constitutes an important modulator of vascular smooth muscle contraction [5-8]. Rho-kinase and its downstream effector are important mediators of not only vascular contraction but also actin cytoskeleton reorganization $[9,10]$, cellular morphology [11], motility [12], adhesion, and proliferation [5-8]. Due to its effects on various cellular functions, Rho-kinase has attracted significant interest as a potential target for the treatment of a wide range of pathological conditions including cancer, neuronal degeneration, kidney failure, asthma, glaucoma, osteoporosis, erectile dysfunction, and surgical adhesion [13-15]. Recent studies have shown that the Rho/Rho-kinase pathway is associated with tissue fibrosis and inflammation. It has also been 
demonstrated that the Rho/Rho-kinase pathway is involved in the tissue fibrosis process through regulation of TGF- $\beta$ activation [16-18].

Therefore, we hypothesized that Y-27632 could be used as an adjuvant to suppress inflammation and fibrosis after strabismus surgery. The present study was conducted to investigate the effect of Y-27632 on postoperative inflammation and fibrosis following extraocular muscle surgery in a rabbit model.

\section{Materials and Methods}

2.1. Rabbits. In total, 16 eyes from 8 New Zealand white rabbits (each weighing 2.0-3.0 kg, 20 weeks old) were used for this study. All rabbits had anatomically normal eyes. All experiments were conducted in accordance with the tenets of the ARVO Statement for the Use of Animals in Ophthalmic and Vision Research. The protocol followed the guidelines of the Declaration of Helsinki and was approved by the Institutional Review Board of The Catholic University of Korea, College of Medicine.

2.2. Surgical Procedures. Each rabbit was anesthetized with intramuscular xylazine hydrochloride $(5 \mathrm{mg} / \mathrm{kg})$ and ketamine hydrochloride $(40 \mathrm{mg} / \mathrm{kg})$ and received a topical anesthetic containing $0.5 \%$ proparacaine hydrochloride (Alcaine $0.5 \%$; Alcon, USA).

In both eyes of each rabbit, the superior conjunctiva and Tenon's capsule were opened, and the superior rectus muscle (SRM) was exposed using cotton swabs and a muscle hook. The SRM was then placed on a double-armed 6-0 Vicryl (polyglactin) suture that was located near the insertion site and detached from the globe. The SRM was sutured back to its original insertion after detachment, and the conjunctival suturing was performed with an interrupted 8-0 Vicryl suture.

In the right eye (treated eye) of each rabbit, $0.2 \mathrm{~mL}$ of $10 \mu \mathrm{M}$ Y-27632 was injected; $0.2 \mathrm{~mL}$ saline was injected into the left eye (control eye) subconjunctivally. To prevent infection, ofloxacin eye drops (Tarivid, Santen, Osaka, Japan) were applied once on each eye after surgery.

Rabbits were randomly selected for sacrifice using barbiturate anesthesia in a group of 4 ( 8 eyes) at postoperative day 3 and week 4 . All eyes were enucleated and examined.

2.3. Evaluation of Inflammation. After animal sacrifice at postoperative day 3 and week 4 , the sites of muscle reattachment were fixed in $10 \%$ formalin for $24 \mathrm{~h}$, stored in $70 \%$ alcohol, and embedded in paraffin. Sequential $5 \mu \mathrm{m}$ sections of the wound site were prepared. Sections were examined microscopically using hematoxylin and eosin (H\&E) staining and immunofluorescence. The sections were examined using rabbit polyclonal antibodies for Ki-67, CD11 $\beta+$, and F4/80. The antibody-positive cell staining level was graded by a masked observer based on a histologic grading scale of 1 to $4(1=0-10 \%, 2=10-30 \%, 3=30-50 \%$, and $4=>50 \%$ antibody-positive cell density).

2.4. Assessment of Late Adhesion and Fibrosis. Both eyes from 4 rabbits were examined regarding their gross adhesion score to evaluate postoperative fibrosis 4 weeks after the operation. Adhesion score severities were from 0 to 3 according to the criteria in a previous report, where 0 indicated no adhesion, 1 indicated adhesion easily separated with blunt dissection, 2 indicated mild-to-moderate adhesion with the freely dissectible plane, and 3 indicated moderate-to-dense adhesion with difficult dissection or a nondissectable plane [19].

Sections were also stained using Masson's trichrome staining kit (HT15, Sigma-Aldrich, St. Louis, MO, USA) and a fibronectin staining kit for quantification of collagen. Fibrosis was graded according to the amount of collagen as follows: 0 , no fibrosis; 1 , mild perimuscular fibrotic reaction (stained collagen was detectable only in thin bands immediately adjacent to muscle); 2 , easily detected thick bands; 3 , well-developed, dense bands of collagen; and 4, severe fibrotic response replacing large areas [20].

2.5. Statistical Analysis. All statistical analyses were performed using the SPSS software (ver. 17.0; SPSS, Chicago, IL, USA). Wilcoxon signed-rank tests were applied to make the comparison between the two postoperative groups. Values of $p<0.05$ were considered statistically significant.

\section{Results}

During the follow-up period, all rabbits survived and remained in good health until sacrifice. No severe systemic complications were observed in any of the animals.

3.1. Acute Inflammation. Histologic examination by $\mathrm{H} \& \mathrm{E}$ staining showed inflammatory cell infiltration around the SRM and surrounding tissue in both groups.

Immunofluorescence of $\mathrm{Ki}-67$ and $\mathrm{CD} 11 \beta+$ was not significantly different in the Y-27632 injection group compared to the control at 3 days $(p=0.500,0.054)$ and 4 weeks $(p=0.235,0.692)$, respectively, postoperatively. However, F4/80 antibody expression was significantly different in the Y-27632 injection group at both 3 days and 4 weeks postoperatively $(p=0.038,0.031)$ (Figures 1 and 2 ).

3.2. Late Fibrosis. The SRM/conjunctiva adhesion score differed significantly between the groups $(p=0.034)$. However, the SRM/sclera adhesion score did not differ significantly between the groups $(p=0.178) 4$ weeks after extraocular muscle surgery.

$\mathrm{H} \& \mathrm{E}$ staining revealed some degree of granulation tissue with fibrosis around the SRM in both groups. Masson's trichrome stain in the SRM at postoperative 4 weeks presented some degree of blue-colored collagen in the endomysium in both groups. In addition, fibronectin stain in the SRM at postoperative 4 weeks presented some degree of red-colored tissues. The degrees of fibrosis in both stainings were lower in the Y-27632 injection group compared to the control, but not so significant (Figures 3(a) and 3(b)).

In addition, the muscle fibers were more highly separated by collagen deposition in the control group. With regard to postoperative changes, some muscle fibers were stained red faintly in the control group, whereas most were stained red distinctly in the Y-27632 injection group. 


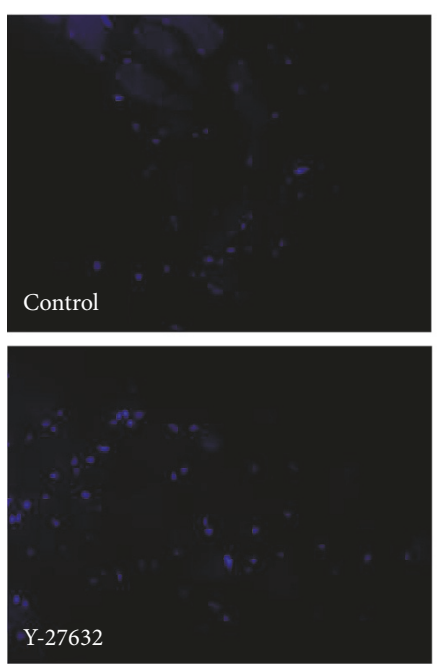

DAPI
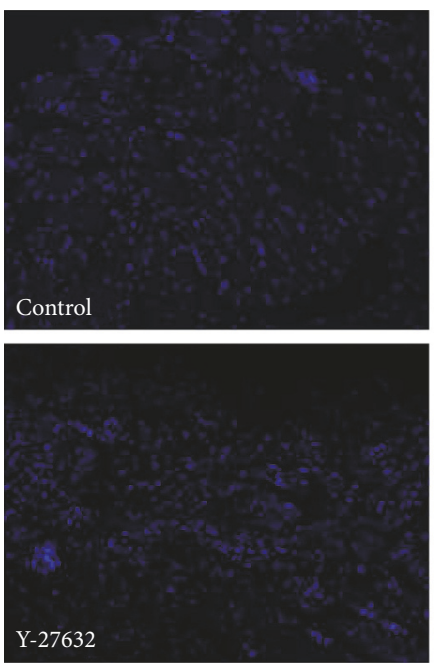

DAPI
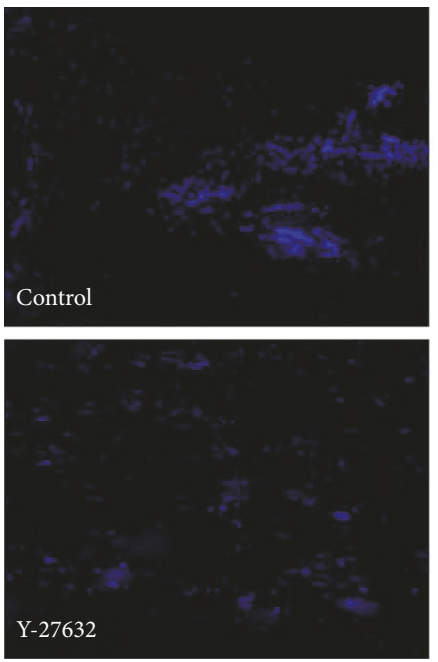

DAPI
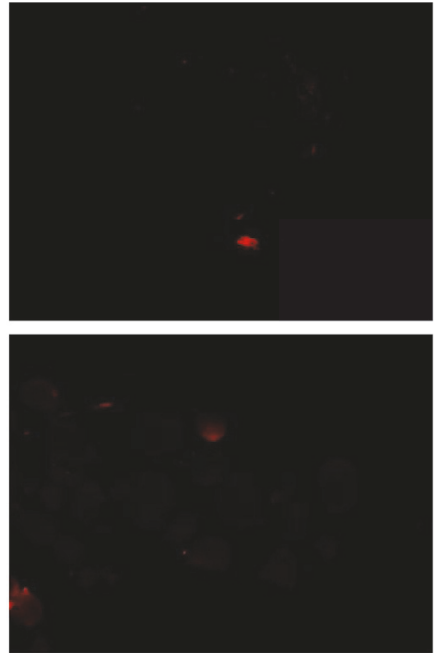

Ki-67

(a)
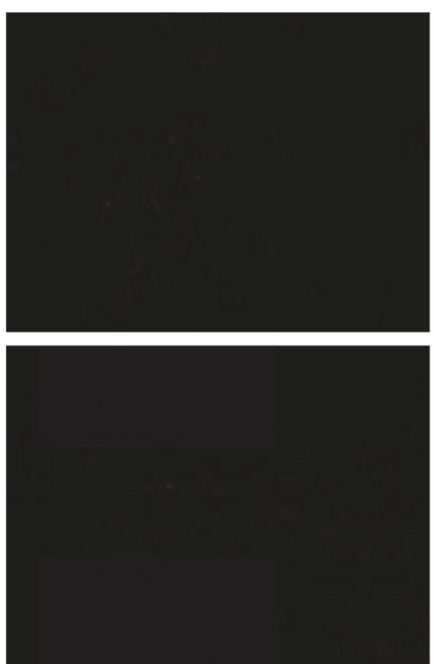

CD11b

(b)
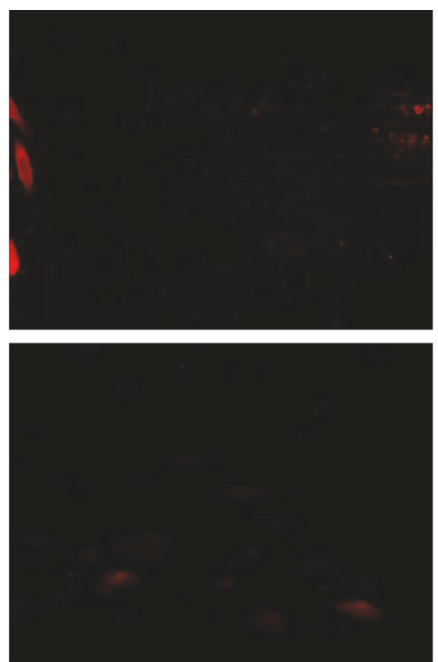

F4/80

(c)
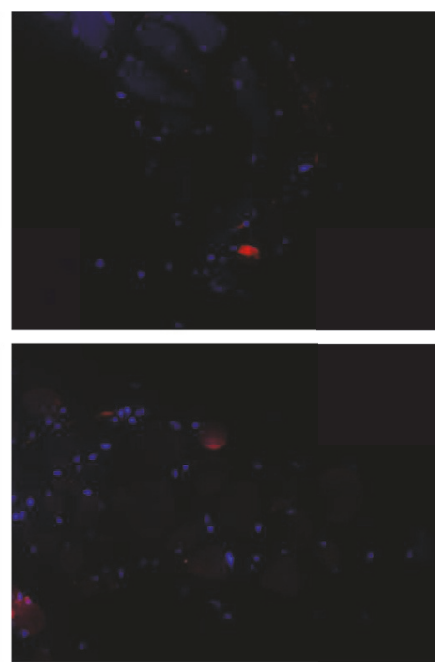

Merged
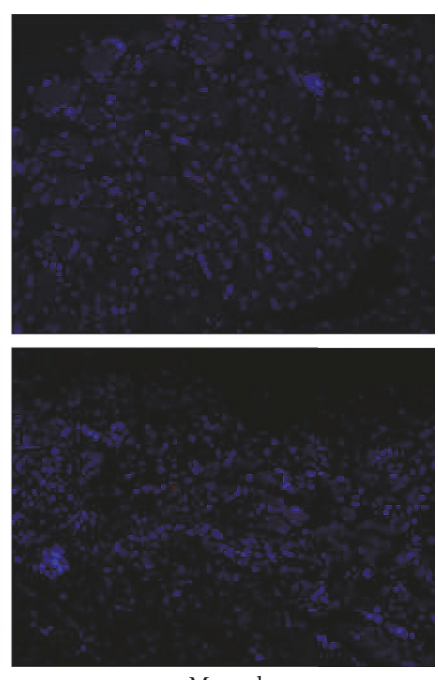

Merged
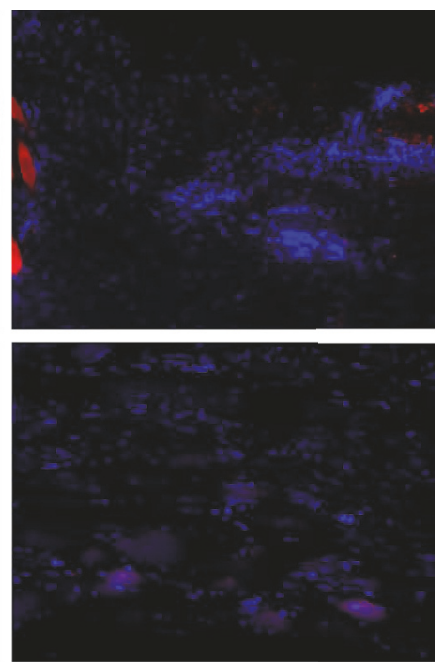

Merged

Figure 1: Continued. 


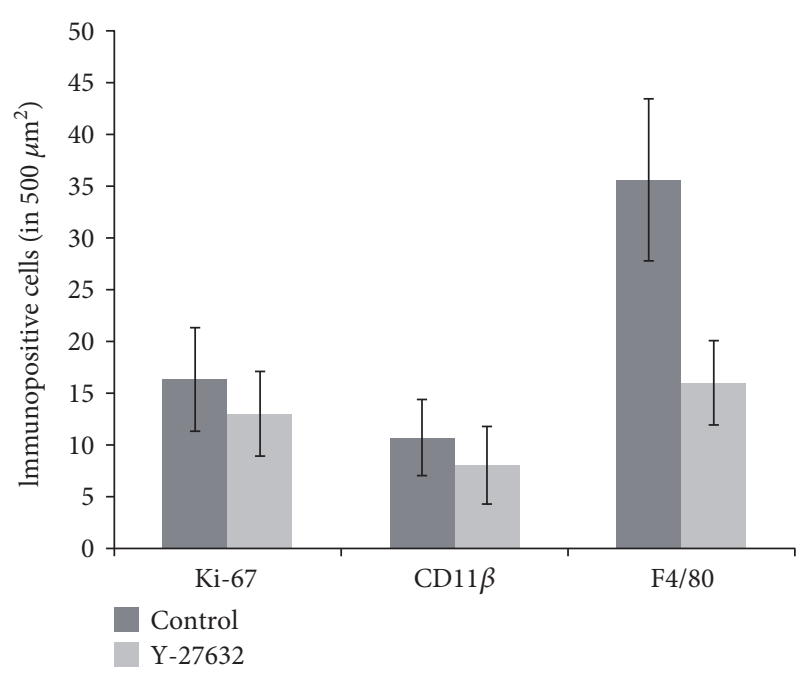

(d)

FiguRE 1: Immunohistochemical staining for Ki-67 (a), CD11 beta (b), and F4/80 (c) of the superior rectus muscle (SRM) 3 days after reinsertion of the SRM in a rabbit model: the normal saline injection group (above) and the Y-27632 injection group (below). Compared to the control group in which saline was injected into the SRM, only F4/80-positive areas were less present in the Y-27632 injection group (magnification, $\times 100$ ). Immunepositive cells of Ki-67, CD11 beta, and F4/80 at 3 days after reinsertion of the SRM in a rabbit model (d).

\section{Discussion}

In the present study, we demonstrated that the inhibitor of Rho-kinase, Y-27632, was able to reduce inflammation and SRM/conjunctiva adhesion in rabbit eyes following extraocular muscle surgery. Our results show that immunofluorescence staining of F4/80 decreased at postoperative day 3 and week 4. F4/80, a macrophage marker [21], exhibited lower positivity in the Y-27632 injection group compared to the control, implying that Y-27632 decreased the number of macrophages recruited to the wound site; that is, Y-27632 may suppress the macrophage-mediated inflammatory response. The protein serine/threonine kinase ROCK1 is the main effector of Rho GTPase RhoA, and the RhoA/ ROCK pathway is the main regulator of actin cytoskeleton and actin-related functions in the cells. During the immune response, the movement of macrophages toward the target relies on proper organization of actin cytoskeleton and focal adhesions in the front and back of the cell. Y-27632 is a recently identified specific inhibitor of the ROCK-ROK family of protein kinases. Inhibition of ROCK1 by Y27632 abolishes macrophage polarity and reduces their podosomes, motility, and phagocytosis and increases matrix degradation [22-25].

One of the predominant inflammatory cells within a wound is the macrophage at 48 to $96 \mathrm{~h}$ after wounding. Macrophages act as a major source of various cytokines and growth factors and are required to support cellular recruitment and activation, matrix synthesis, angiogenesis, and remodeling. Unlike neutrophils, macrophages remain within a wound until healing is complete [26].

Immunofluorescence staining of F4/80 was decreased in the Y-27632 injection group, but an expression of CD11 $\beta$ and Ki-67 at postoperative day 3 and week 4 was not different between the groups. CD11 $\beta$ is expressed on the surface of many leukocytes involved in the innate immune system, including monocytes, granulocytes, macrophages, and natural killer cells [27]. Additionally, Ki-67 is a cellular marker of proliferation [28] with which it is strictly associated. Our results imply that Y-27632 injection may decrease the inflammatory response mediated by macrophages, but this does not occur by either inhibition of leukocyte recruitment or inflammatory cell proliferation. Our study shows only the results of postoperative day 3 and week 4 . Therefore, further studies are needed to demonstrate the intermediate process in order to confirm that the results of three days last up to four weeks.

Macrophages and fibroblasts release numerous growth factors and cytokines that contribute to fibroblast migration: fibroblast growth factor (FGF), IGF-1, vascular endothelial growth factor (VEGF), IL-1, IL-2, IL-8, plateletderived growth factor (PDGF), TGF- $\alpha$, TGF- $\beta$, and TNF- $\alpha$. Fibroblasts are activated to proliferate and begin synthesizing collagen. These activated fibroblasts are related to adhesion and fibrosis [26].

In this study, the SRM/conjunctiva adhesion score differed between the groups; this might be interpreted as a reduction in the macrophage-mediated inflammatory response, thus reducing adhesion and fibrosis. However, the SRM/sclera adhesion score and Masson's trichrome/fibronectin staining did not differ significantly. Therefore, we believe that other factors in addition to macrophages might be involved in the inflammatory process and the activation of fibroblasts in the extraocular muscle and surrounding connective tissue. Analysis of the tissue should consider the possibility of biases due to lengthened scars, consisting of amorphous connective tissue. Therefore, all procedures of this study were conducted by a single ophthalmologist who had experiences of strabismus operation more than ten years. In addition, factors that distinguish stretched scars from 

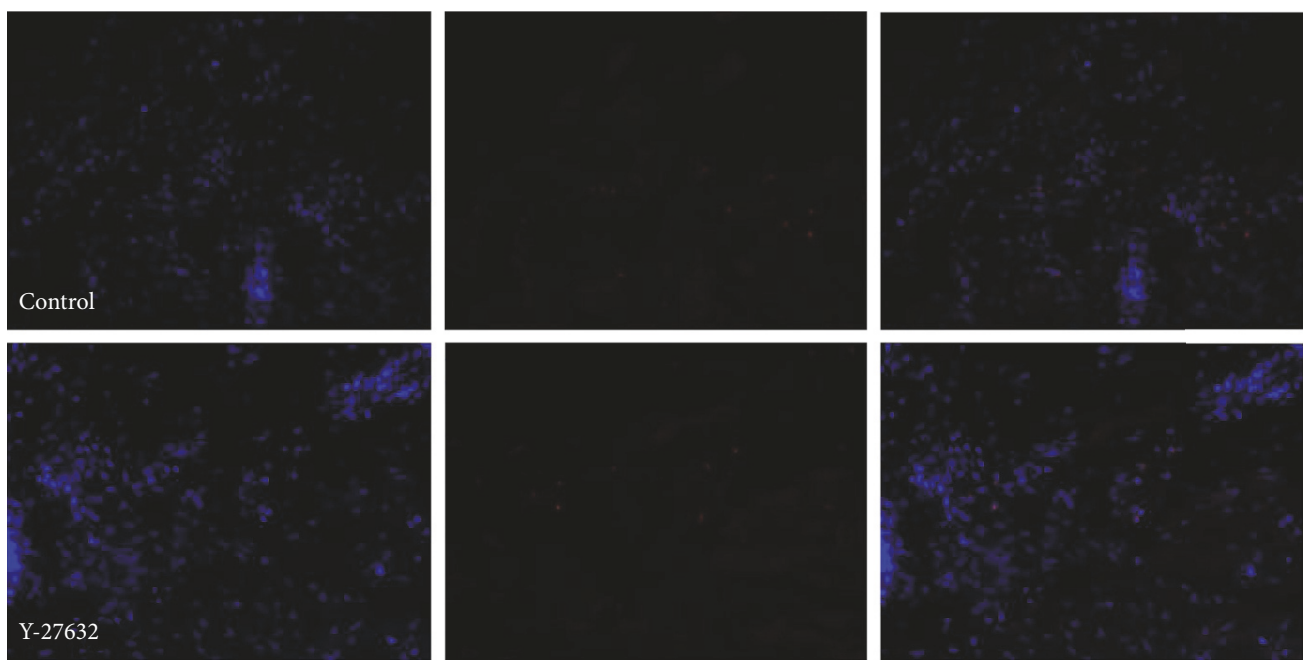

DAPI

Merged

(a)
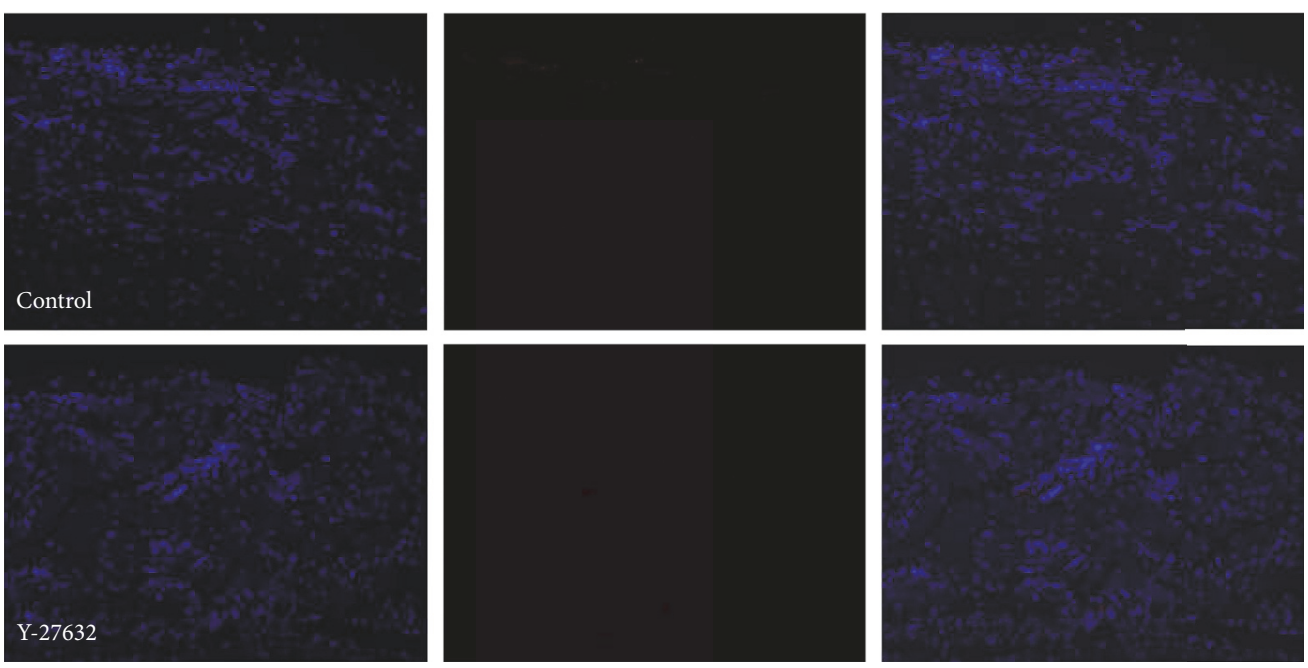

DAPI

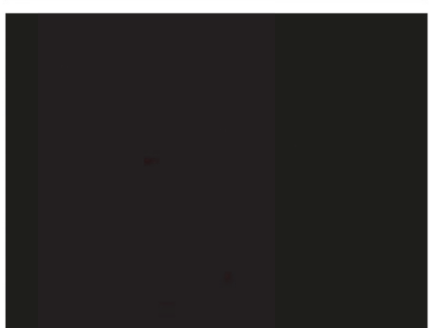

CD11b

(b)
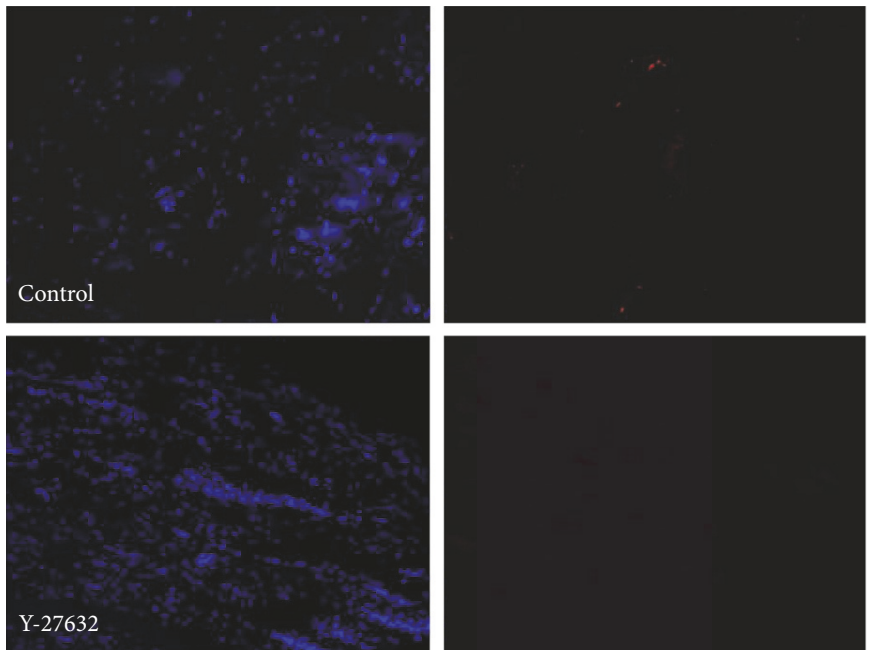

DAPI

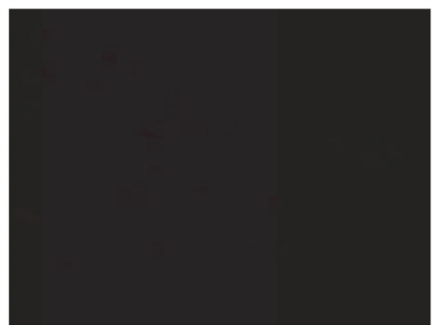

$\mathrm{F} 4 / 80$

(c)
Merged
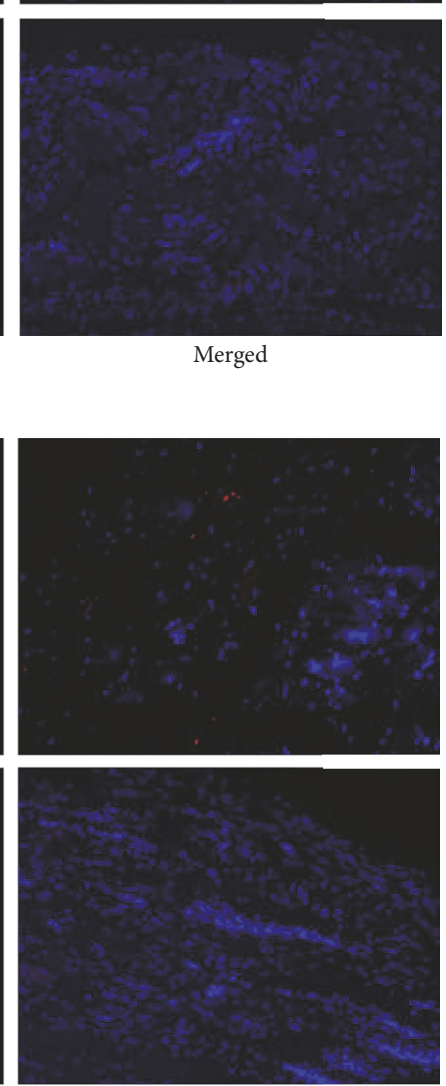

Merged

Figure 2: Continued. 


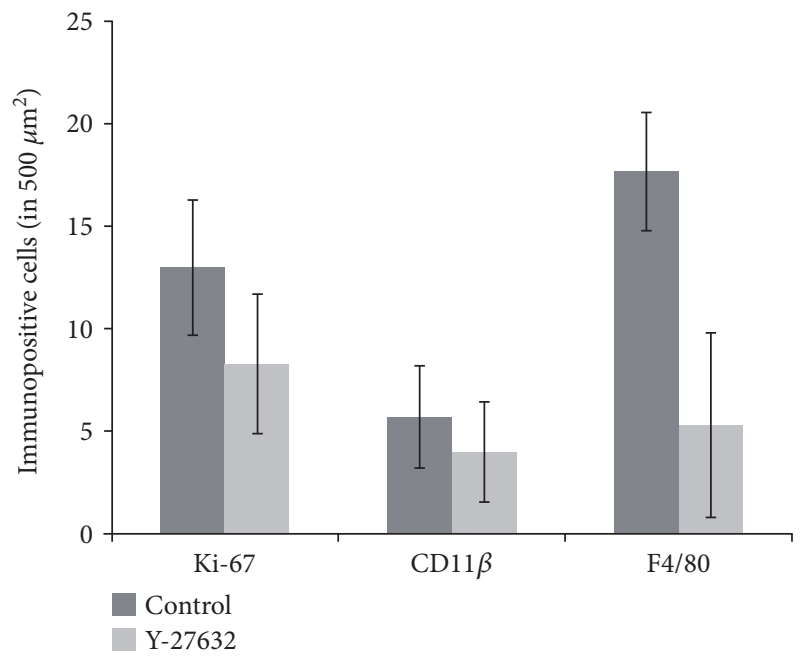

(d)

FIGURE 2: Immunohistochemical staining for Ki-67 (a), CD11 beta (b), and F4/80 (c) of the SRM 4 weeks after reinsertion of the SRM in a rabbit model: the normal saline injection group (above) and the Y-27632 injection group (below). Compared to the control group in which saline was injected into the SRM, only F4/80-positive areas were less present in the Y-27632 injection group (magnification, $\times 100$ ). Immunepositive cells of Ki-67, CD11 beta, and F4/80 at 4 weeks after reinsertion of the SRM in a rabbit model (d).
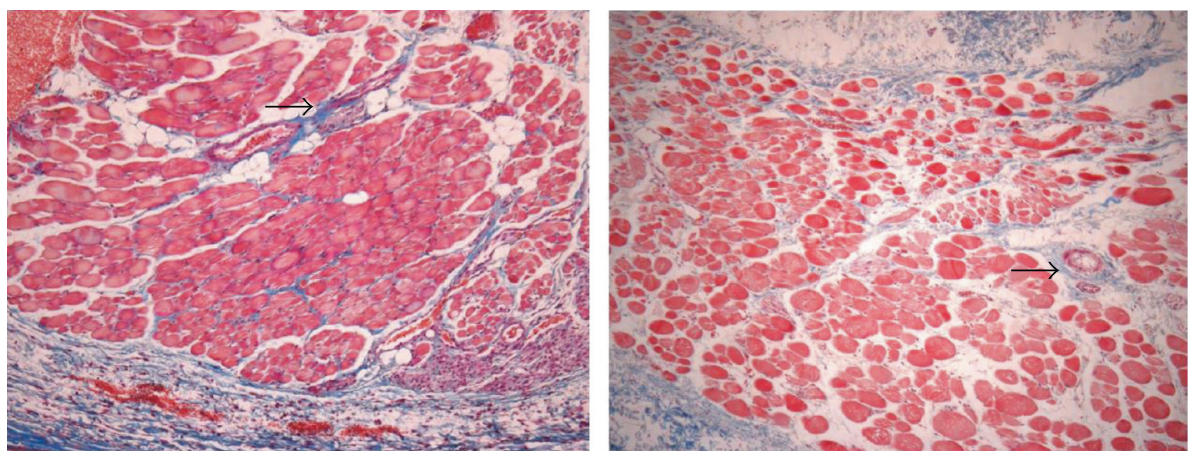

(a)
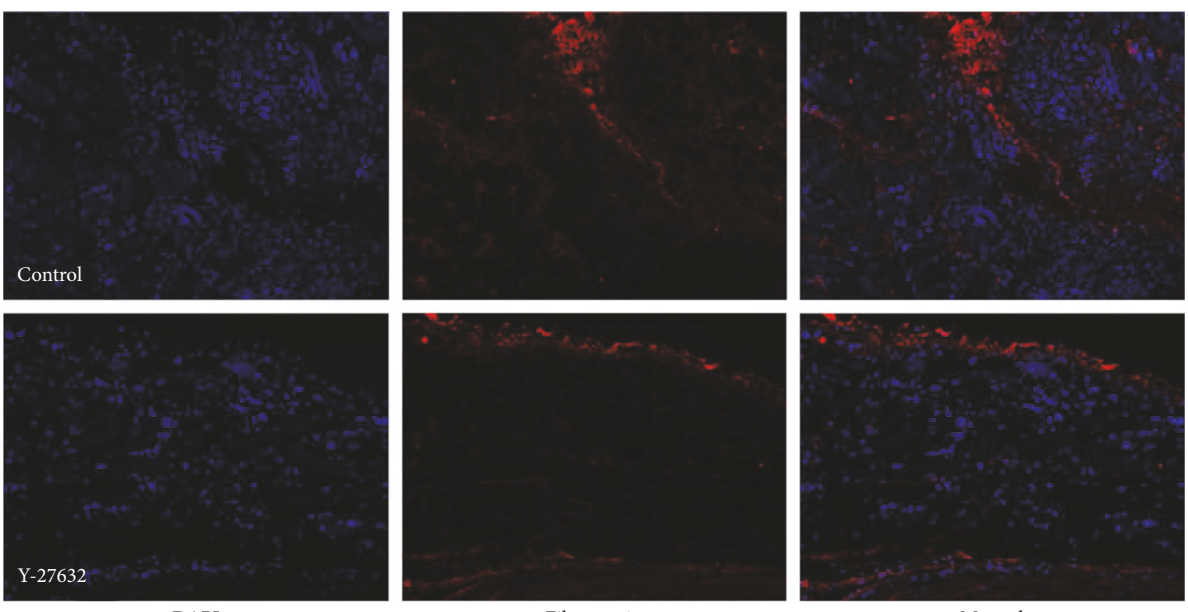

DAPI

Fibronectin

Merged

(b)

FIGURE 3: Light microscopy findings (Masson trichrome; original magnification, $\times 100$ ) of the SRM 4 weeks after surgery (a): the normal saline injection group (left) and the Y-27632 injection group (right). Noted fibrosis within the muscle bundles was observed in the control group. Some muscle fiber was stained red in addition to the blue color (arrow). Mild fibrosis was observed in the Y-27632 group. Light microscopy findings (fibronectin; original magnification, $\times 100$ ) of the SRM 4 weeks after surgery (b): the normal saline injection group (above) and the Y27632 injection group (below). 
classic slipped muscles included minimal or no limitation of versions, less separation of the tendons from the sclera, and thicker appearance of the scar segments [29].

Rho-GTPase participates in signaling pathways leading to the formation of actin stress fibers and focal adhesions. Rho is also involved in diverse physiological functions associated with cytoskeletal rearrangements, such as cell morphology, cell motility, cytokinetics, and smooth muscle contraction. Rho-associated protein kinases (ROCKs) play a key role in focal adhesions and stress fiber formation in cultured fibroblasts and epithelial cells and in $\mathrm{Ca}^{+2}$ sensitization of smooth muscle cells [30]. Y-27632 is a recently identified specific inhibitor of the ROCK-ROK family of protein kinases. Y-27632 inhibits $\mathrm{Ca}^{+2}$-sensitive smooth muscle contraction and has been suggested as a useful therapeutic for hypertension [13].

Recent studies have shown that the Rho/Rho-kinase pathway is associated with tissue fibrosis and inflammation [31-34]. The Rho/ROCK-mediated pathway plays a role in the infiltration of inflammatory cells both in vitro and in vivo $[16,17]$. Y-27632 also prevented the upregulation of $\alpha$-smooth muscle actin ( $\alpha$-SMA), a marker of tissue fibrosis, and inhibited tubulointerstitial fibrosis in mouse kidney with unilateral ureteral obstruction [14]. Another study reported that the selective ROCK inhibitor, fasudil, had beneficial effects on bleomycin-induced pulmonary fibrosis in mice. The number of infiltrated inflammatory cells in bronchoalveolar lavage fluid (BALF) was attenuated by fasudil [32]. The present results suggest that Y-27632 inhibits the macrophage-mediated inflammatory response and reduces adhesion and fibrosis.

In conclusion, our data suggest that injection of Y-27632 inhibited inflammation and adhesion during the wound healing process. Y-27632 may thus have significant potential as prophylaxis for postoperative adhesion syndrome in extraocular muscle surgery.

\section{Disclosure}

This paper was presented as a conference paper in the 10th ISOPT (International Symposium on Ocular Pharmacology and Therapeutics) Clinical.

\section{Conflicts of Interest}

All authors have completed and submitted the ICMJE form for disclosure of potential conflicts of interest.

\section{Authors' Contributions}

Sun Young Shin designed the study. Hyun Kyung Kim and Sun Young Shin conducted the study. Hyun Kyung Kim and Sun Young Shin contributed to the collection, management, analysis, and interpretation of the data. Ji-Sun Moon and Sun Young Shin prepared the manuscript. Ji-Sun Moon and Sun Young Shin reviewed and approved the manuscript.

\section{References}

[1] N. Sondhi, F. D. Ellis, L. M. Hamed, and E. M. Helveston, "Evaluation of an absorbable muscle sleeve to limit postoperative adhesions in strabismus surgery," Ophthalmic Surgery, vol. 18, no. 6, pp. 441-443, 1987.

[2] E. S. Guclu, A. A. Sari, E. Dinc, T. Ozcan Metin, B. Coskun Yilmaz, and B. Tasdelen, "Efficacy of mitomycin-C and infliximab in reducing adhesion and fibrosis following strabismus surgery," Turkish Journal of Medical Sciences, vol. 46, no. 5, pp. 1401-1406, 2016.

[3] S. Choi, Y. Cheong, J. H. Shin et al., "Short-term response of mitomycin $\mathrm{C}$ on the human rectus muscle following strabismus surgery: histological, ultrastructural, and biomechanical evaluation," Microscopy and Microanalysis, vol. 19, no. 1, pp. 227-232, 2013.

[4] M. P. Diamond, E. L. Burns, B. Accomando, S. Mian, and L. Holmdahl, "Seprafilm(R) adhesion barrier: (1) a review of preclinical, animal, and human investigational studies," Gynecological Surgery, vol. 9, no. 3, pp. 237-245, 2012.

[5] C. Nobes and A. Hall, "Regulation and function of the Rho subfamily of small GTPases," Current Opinion in Genetics \& Development, vol. 4, no. 1, pp. 77-81, 1994.

[6] Y. Takai, T. Sasaki, K. Tanaka, and H. Nakanishi, "Rho as a regulator of the cytoskeleton," Trends in Biochemical Sciences, vol. 20, no. 6, pp. 227-231, 1995.

[7] A. J. Ridley, H. F. Paterson, C. L. Johnston, D. Diekmann, and A. Hall, "The small GTP-binding protein rac regulates growth factor-induced membrane ruffling," Cell, vol. 70, no. 3, pp. 401-410, 1992.

[8] A. J. Ridley and A. Hall, "Signal transduction pathways regulating Rho-mediated stress fibre formation: requirement for a tyrosine kinase," The EMBO Journal, vol. 13, no. 11, pp. 2600-2610, 1994.

[9] S. Narumiya, T. Ishizaki, and N. Watanabe, "Rho effectors and reorganization of actin cytoskeleton," FEBS Letters, vol. 410, no. 1, pp. 68-72, 1997.

[10] D. Thumkeo, S. Watanabe, and S. Narumiya, "Physiological roles of Rho and Rho effectors in mammals," European Journal of Cell Biology, vol. 92, no. 10-11, pp. 303-315, 2013.

[11] H. F. Paterson, A. J. Self, M. D. Garrett, I. Just, K. Aktories, and A. Hall, "Microinjection of recombinant p21rho induces rapid changes in cell morphology," The Journal of Cell Biology, vol. 111, no. 3, pp. 1001-1007, 1990.

[12] K. Takaishi, T. Sasaki, M. Kato et al., "Involvement of Rho p21 small GTP-binding protein and its regulator in the HGF-induced cell motility," Oncogene, vol. 9, no. 1, pp. 273279, 1994.

[13] M. F. Olson, "Applications for ROCK kinase inhibition," Current Opinion in Cell Biology, vol. 20, no. 2, pp. 242248, 2008.

[14] K. Nagatoya, T. Moriyama, N. Kawada et al., "Y-27632 prevents tubulointerstitial fibrosis in mouse kidneys with unilateral ureteral obstruction," Kidney International, vol. 61, no. 5, pp. 1684-1695, 2002.

[15] Y. Feng, P. V. LoGrasso, O. Defert, and R. Li, "Rho kinase (ROCK) inhibitors and their therapeutic potential," Journal of Medicinal Chemistry, vol. 59, no. 6, pp. 2269-2300, 2016.

[16] V. Niggli, "Rho-kinase in human neutrophils: a role in signalling for myosin light chain phosphorylation and cell migration," FEBS Letters, vol. 445, no. 1, pp. 69-72, 1999. 
[17] Y. Shimizu, K. Dobashi, K. Iizuka et al., "Contribution of small GTPase Rho and its target protein rock in a murine model of lung fibrosis," American Journal of Respiratory and Critical Care Medicine, vol. 163, no. 1, pp. 210-217, 2001.

[18] D. Warburton, W. Shi, and B. Xu, "TGF-beta-Smad3 signaling in emphysema and pulmonary fibrosis: an epigenetic aberration of normal development?" American Journal of Physiology - Lung Cellular and Molecular Physiology, vol. 304, no. 2, pp. L83-L85, 2013.

[19] Y. Yaacobi, L. M. Hamed, K. S. Kaul, and M. M. Fanous, "Reduction of postoperative adhesions secondary to strabismus surgery in rabbits," Ophthalmic Surgery, vol. 23, no. 2, pp. 123-128, 1992.

[20] J. S. Mora, D. T. Sprunger, E. M. Helveston, and A. P. Evan, "Intraoperative sponge 5-fluorouracil to reduce postoperative scarring in strabismus surgery," Journal of AAPOS, vol. 1, no. 2, pp. 92-97, 1997.

[21] P. J. Leenen, M. F. de Bruijn, J. S. Voerman, P. A. Campbell, and W. van Ewijk, "Markers of mouse macrophage development detected by monoclonal antibodies," Journal of Immunological Methods, vol. 174, no. 1-2, pp. 5-19, 1994.

[22] Y. Liu, N. Tejpal, J. You, X. C. Li, R. M. Ghobrial, and M. Kloc, "ROCK inhibition impedes macrophage polarity and functions," Cellular Immunology, vol. 300, pp. 54-62, 2016.

[23] K. M. Bourzac, C. M. Botham, and K. Guillemin, "Helicobacter pylori CagA induces AGS cell elongation through a cell retraction defect that is independent of Cdc42, Rac1, and Arp2/3," Infection and Immunity, vol. 75, no. 3, pp. 1203-1213, 2007.

[24] E. C. Lessey, C. Guilluy, and K. Burridge, "From mechanical force to RhoA activation," Biochemistry, vol. 51, no. 38, pp. 7420-7432, 2012.

[25] M. Vicente-Manzanares, D. J. Webb, and A. R. Horwitz, "Cell migration at a glance," Journal of Cell Science, vol. 118, no. 21, pp. 4917-4919, 2005.

[26] P. Teller and T. K. White, "The physiology of wound healing: injury through maturation," The Surgical Clinics of North America, vol. 89, no. 3, pp. 599-610, 2009.

[27] D. A. Solovjov, E. Pluskota, and E. F. Plow, "Distinct roles for the alpha and beta subunits in the functions of integrin alphaMbeta2," The Journal of Biological Chemistry, vol. 280, no. 2, pp. 1336-1345, 2005.

[28] T. Scholzen and J. Gerdes, "The Ki-67 protein: from the known and the unknown," Journal of Cellular Physiology, vol. 182, no. 3, pp. 311-322, 2000.

[29] I. H. Ludwig and A. Y. Chow, "Scar remodeling after strabismus surgery," Journal of AAPOS, vol. 4, no. 6, pp. 326-333, 2000.

[30] M. Uehata, T. Ishizaki, H. Satoh et al., "Calcium sensitization of smooth muscle mediated by a Rho-associated protein kinase in hypertension," Nature, vol. 389, no. 6654, pp. 990-994, 1997.

[31] N. Washida, S. Wakino, Y. Tonozuka et al., "Rho-kinase inhibition ameliorates peritoneal fibrosis and angiogenesis in a rat model of peritoneal sclerosis," Nephrology, Dialysis, Transplantation, vol. 26, no. 9, pp. 2770-2779, 2011.

[32] C. Jiang, H. Huang, J. Liu, Y. Wang, Z. Lu, and Z. Xu, "Fasudil, a Rho-kinase inhibitor, attenuates bleomycininduced pulmonary fibrosis in mice," International Journal of Molecular Sciences, vol. 13, no. 7, pp. 8293-8307, 2012.
[33] J. E. Bond, G. Kokosis, L. Ren, M. A. Selim, A. Bergeron, and H. Levinson, "Wound contraction is attenuated by fasudil inhibition of Rho-associated kinase," Plastic and Reconstructive Surgery, vol. 128, no. 5, pp. 438e-450e, 2011.

[34] X. J. Qi, W. Ning, F. Xu, H. X. Dang, F. Fang, and J. Li, "Fasudil, an inhibitor of Rho-associated coiled-coil kinase, attenuates hyperoxia-induced pulmonary fibrosis in neonatal rats," International Journal of Clinical and Experimental Pathology, vol. 8, no. 10, pp. 12140-12150, 2015. 


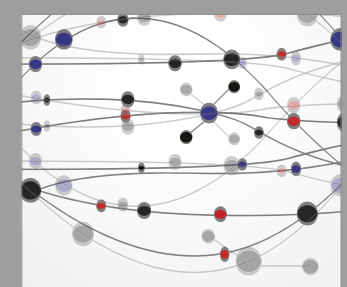

The Scientific World Journal
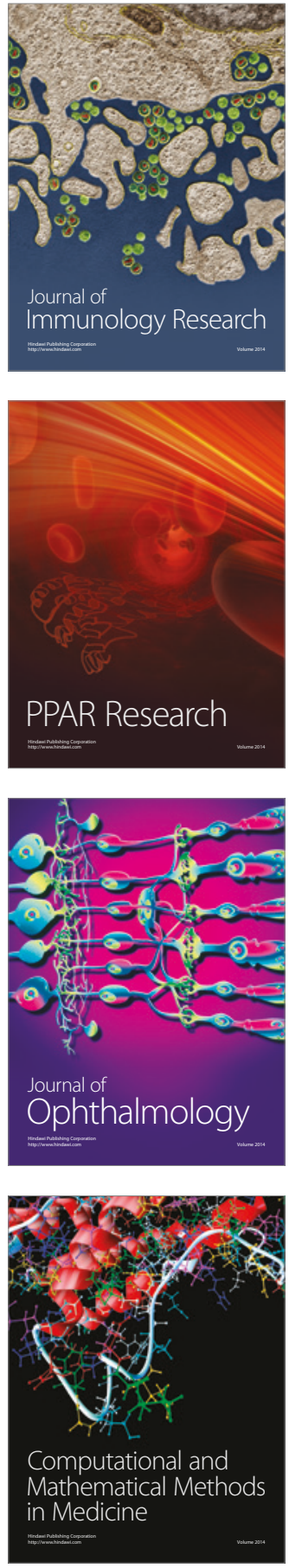

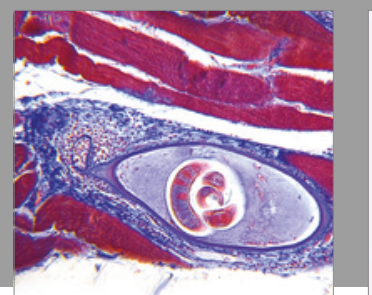

Gastroenterology Research and Practice
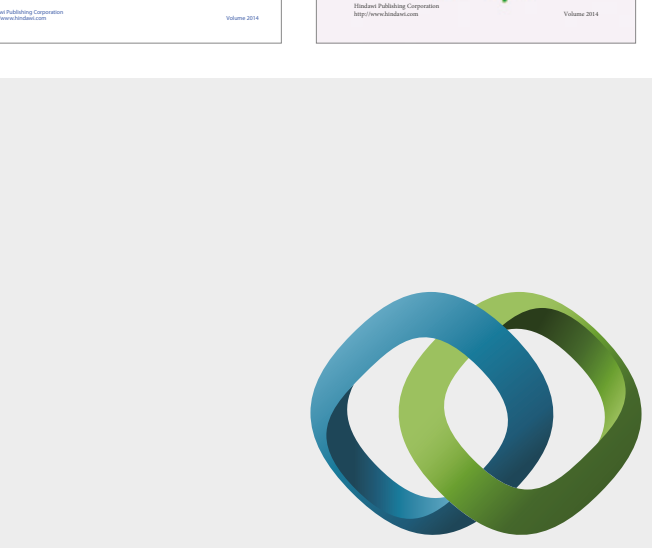

\section{Hindawi}

Submit your manuscripts at

https://www.hindawi.com
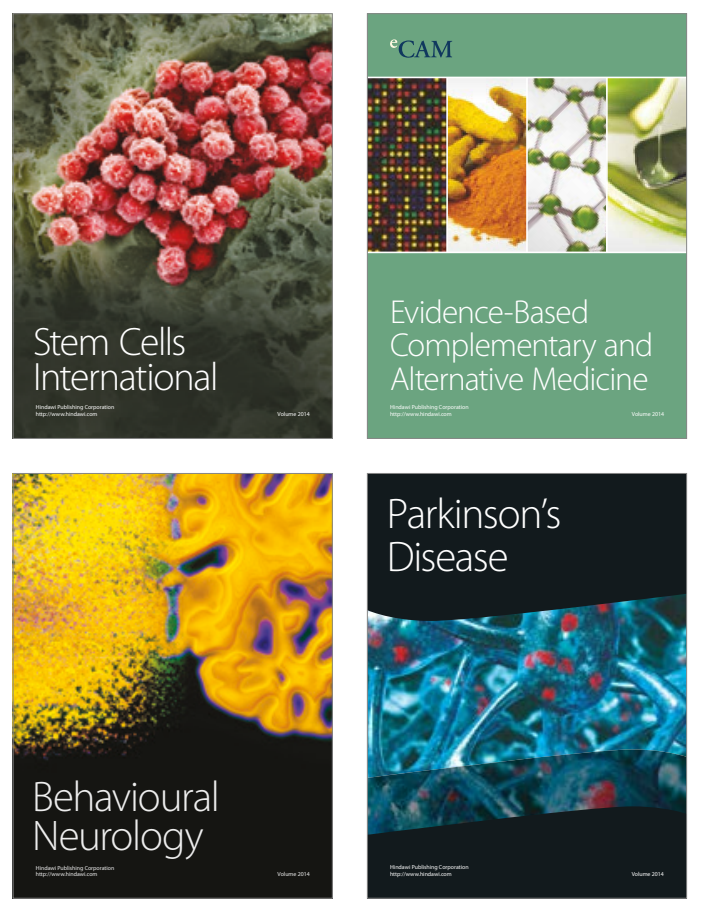
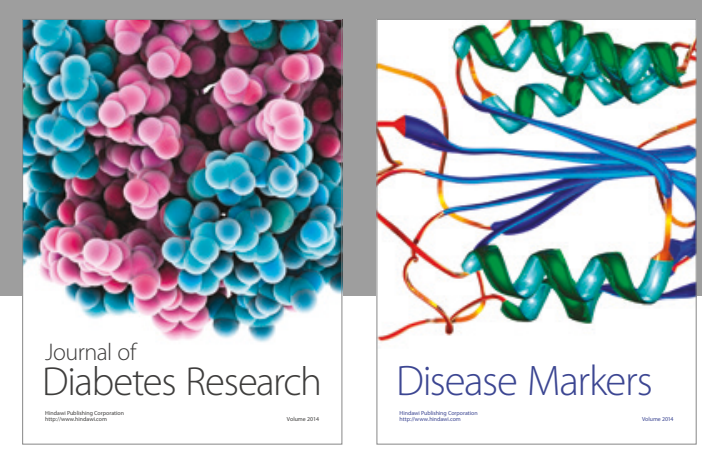

Disease Markers
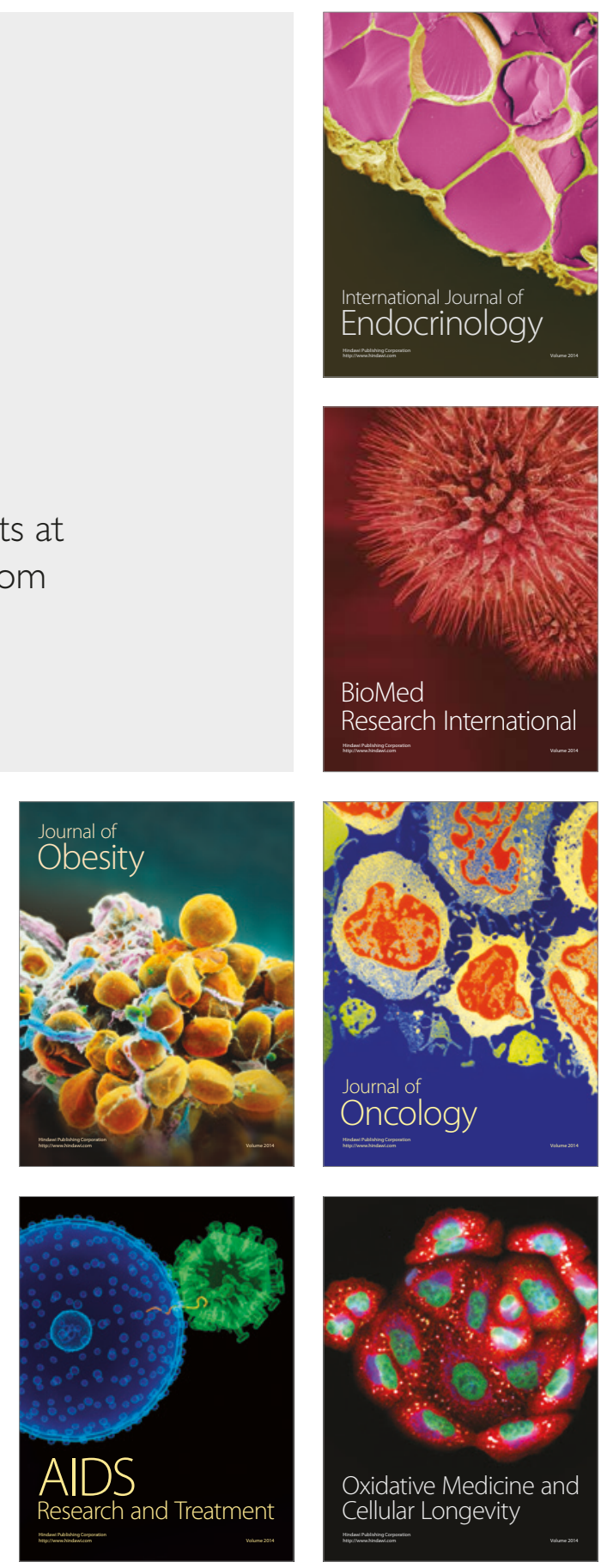